\title{
Solvability and Galerkin Approximations of a Class of Nonlinear Operator Equations
}

\author{
G. N. Gatica
}

\begin{abstract}
We generalize the usual Babuška-Brezzi theory to a class of nonlinear variational problems with constraints. The corresponding operator equation has a dual-dual type structure since the nonlinear operator involved has itself a dual structure with a strongly monotone and Lipschitz-continuous main operator. We provide sufficient conditions for the existence and uniqueness of solution of the continuous and Galerkin formulations, and derive a Strang-type estimate for the associated error. An application to the coupling of mixedFEM and BEM for a nonlinear transmission problem in potential theory is also described.

Keywords: Dual-dual formulation of problems, extended Babuška-Brezzi theory, inf-sup conditions, strongly monotone operators
\end{abstract}

AMS subject classification: $65 \mathrm{~J} 15,65 \mathrm{~N} 30,65 \mathrm{~N} 38,65 \mathrm{R} 20$

\section{Introduction}

The classical Babuška-Brezzi theory for variational problems with constraints is a very well established tool for studying the weak solutions and Galerkin approximations, via mixed formulations, of a wide class of linear boundary value problems in physics and engineering sciences (see, e.g., $[2,12]$ and the references therein).

However, the corresponding theory for nonlinear problems has not been fully developed and only a few fundamental references can be found in the literature. For the case of nonlinear problems with nonlinear constraints, we refer to $[16,17]$, where the abstract theory and its applications to some problems in nonlinear incompressible elasticity are presented. Also, we may mention the work [19] for nonlinear problems with linear constraints, that is, those in which the associated operator has the typical dual form $\left[\begin{array}{cc}A & B^{*} \\ B & O\end{array}\right]$, where $B$ is linear and bounded and $A$ is nonlinear. In there, the author uses the theory of monotone operators to obtain existence, uniqueness and approximation results for a family of strongly nonlinear problems.

Now, when dealing with the variational formulations of some nonlinear transmission problems, via the combined use of mixed finite elements (mixed-FEM) and

Gabrie N. Gatica: Departamento de Ingeniería Matemática, Universidad de Concepción, Casilla 160-C, Concepción, Chile; ggatica@ing-mat.udec.cl

This research was partially supported by Fondecyt-Chile through the FONDAP Program in Applied Mathematics, and by the Dirección de Investigación of the Universidad de Concepción through the Advanced Research Groups Program. 
boundary elements (BEM), we have realized recently that a more suitable way of writing those formulations is in the form of what we call the dual-dual type. This means that the corresponding operator equation can be written in the same matrix structure as above, but where the operator $A$ itself presents also the dual structure, that is, $A=\left[\begin{array}{cc}A_{1} & B_{1}^{*} \\ B_{1} & O\end{array}\right]$, with $B_{1}$ linear and bounded and $A_{1}$ nonlinear. Unfortunately, though very close to the classical dual form, this kind of operator equation has not been studied yet.

Therefore, motivated by the applications to the coupling of mixed-FEM and BEM for interior and exterior nonlinear transmission problems, our purpose in this work is to extend the usual Babuska-Brezzi theory to the class of nonlinear operator equations described in the above section. More precisely, we provide existence, uniqueness and approximation results for the dual-dual mixed variational formulations in which the nonlinear operator $A_{1}$ becomes strongly monotone and Lipschitz continuous in the appropriate spaces. A particular case of the general class studied in this paper was considered in [6]. For a quick glance to our main contributions, we refer the reader to Theorems 2.4, 3.2, 4.1 and to Section 5, below.

The rest of the paper is presented as follows. In Sections 2 and 3 we study the continuous and discrete problems, respectively, and derive sufficient conditions for the existence of unique solutions to both formulations. The error analysis is provided in Section 4. We deduce there a Strang-type estimate that contains consistency terms arising from the fact that the kernel of the discrete analogue of the operator $B$ is not necessarily contained in the kernel of $B$. Finally, an application to the coupling of mixed-FEM and BEM for an exterior nonlinear tranmission problem in potential theory is briefly described in Section 5. For related works on the coupling method we refer to $[1,5,7,10,11]$.

\section{The continuous problem}

Let $X_{1}, M_{1}, M$ be Hilbert spaces and define $X=X_{1} \times M_{1}$. We consider a nonlinear operator $A_{1}: X_{1} \rightarrow X_{1}^{\prime}$, and linear bounded operators

$$
\left.\begin{array}{c}
B: X \rightarrow M^{\prime} \\
B_{1}: X_{1} \rightarrow M_{1}^{\prime}
\end{array}\right\} \quad \text { with transposes }\left\{\begin{array}{c}
B^{*}: M \rightarrow X^{\prime} \\
B_{1}^{*}: M_{1} \rightarrow X_{1}^{\prime},
\end{array}\right.
$$

respectively. With $A_{1}, B_{1}, B_{1}^{*}$ we define a nonlinear operator $A: X \rightarrow X^{\prime}$ as

$$
A(t, \sigma)=\left[\begin{array}{cc}
A_{1} & B_{1}^{*} \\
B_{1} & O
\end{array}\right]\left[\begin{array}{l}
t \\
\sigma
\end{array}\right] \in X^{\prime}=X_{1}^{\prime} \times M_{1}^{\prime}
$$

or, equivalently,

$$
\begin{aligned}
& {[A(t, \sigma),(s, \tau)]_{X^{\prime} \times X}} \\
& \quad=\left[A_{1}(t), s\right]_{X_{1}^{\prime} \times X_{1}}+\left[B_{1}^{*}(\sigma), s\right]_{X_{1}^{\prime} \times X_{1}}+\left[B_{1}(t), \tau\right]_{M_{1}^{\prime} \times M_{1}}
\end{aligned}
$$

for all $(t, \sigma),(s, \tau) \in X$. Hereafter, $[\cdot, \cdot]$ denotes the duality pairing indicated by the corresponding subscript. Then, we are interested in the following nonlinear variational problem: 
Given $(\mathcal{F}, \mathcal{G}) \in X^{\prime} \times M^{\prime}$, find $((t, \sigma), u) \in X \times M$ such that

$$
\left[\begin{array}{cc}
A & B^{*} \\
B & O
\end{array}\right]\left[\begin{array}{c}
(t, \sigma) \\
u
\end{array}\right]=\left[\begin{array}{l}
\mathcal{F} \\
\mathcal{G}
\end{array}\right]
$$

that is

$$
\left.\begin{array}{rl}
{[A(t, \sigma),(s, \tau)]_{X^{\prime} \times X}+\left[B^{*}(u),(s, \tau)\right]_{X^{\prime} \times X}} & =[\mathcal{F},(s, \tau)]_{X^{\prime} \times X} \\
{[B(t, \sigma), v]_{M^{\prime} \times M}} & =[\mathcal{G}, v]_{M^{\prime} \times M}
\end{array}\right\}
$$

for all $((s, \tau), v) \in X \times M$.

In what follows, we adapt the analysis from [12: Chapter I/Section 4] to the present situation. To this end, for each $\mathcal{G} \in M^{\prime}$ we set

$$
V(\mathcal{G})=\{(s, \tau) \in X: B(s, \tau)=\mathcal{G}\}
$$

and, in particular,

$$
V=V(O)=\{(s, \tau) \in X: B(s, \tau)=O\}
$$

or, equivalently, $V=\operatorname{Ker}(B)$. Since $B$ is bounded, $V$ becomes a closed subspace of $X$. Also, let $\Pi: X^{\prime} \rightarrow V^{\prime}$ be the canonical injection defined by $\Pi(\mathcal{F})=\left.\mathcal{F}\right|_{V}$ for all $\mathcal{F} \in X^{\prime}$. Then with $(2.2)$ we associate the following problem:

Find $(t, \sigma) \in V(\mathcal{G})$ such that $\Pi A(t, \sigma)=\Pi(\mathcal{F})$, that is

$$
[A(t, \sigma),(s, \tau)]_{X^{\prime} \times X}=[\mathcal{F},(s, \tau)]_{X^{\prime} \times X}
$$

for all $(s, \tau) \in V$.

Clearly, if $((t, \sigma), u) \in X \times M$ is a solution of problem $(2.2)$, then $(t, \sigma) \in V(\mathcal{G})$ and $(t, \sigma)$ is a solution of problem $(2.3)$ since for all $(s, \tau) \in V$

$$
\left[B^{*}(u),(s, \tau)\right]_{X^{\prime} \times X}=[B(s, \tau), u]_{M^{\prime} \times M}=0 .
$$

Conversely, if $(t, \sigma) \in V(\mathcal{G})$ is a solution of problem (2.3) and if $B$ satisfies a suitable condition, then there exists $u \in M$ such that $((t, \sigma), u) \in X \times M$ is a solution of problem (2.2). More precisely, we have the following result, which is an analogue of [12: Chapter I/Theorem 4.1].

Theorem 2.1. For each $(\mathcal{F}, \mathcal{G}) \in X^{\prime} \times M^{\prime}$ there exists a unique $((t, \sigma), u) \in$ $X \times M$ solution of problem (2.2) if and only if the following conditions hold:

(i) There exists $\beta>0$ such that

$$
\sup _{0 \neq(s, \tau) \in X} \frac{[B(s, \tau), v]_{M^{\prime} \times M}}{\|(s, \tau)\|_{X}} \geq \beta\|v\|_{M}
$$

for all $v \in M$. 
(ii) For each $(\mathcal{F}, \mathcal{G}) \in X^{\prime} \times M^{\prime}$ there exists a unique $(t, \sigma) \in V(\mathcal{G})$ solution of problem (2.3).

Proof. We prove first that (i) and (ii) are sufficient. In fact, from (ii) we deduce that there exists a unique $(t, \sigma) \in V(\mathcal{G})$ such that $(\mathcal{F}-A(t, \sigma)) \in V^{o}$, where $V^{o}$ is the polar set of $V$ defined by

$$
V^{o}=\left\{\widetilde{\mathcal{F}} \in X^{\prime}:[\widetilde{\mathcal{F}},(s, \tau)]_{X^{\prime} \times X}=0 \text { for all }(s, \tau) \in V\right\} .
$$

Now, by virtue of (i) and [12: Chapter I/Lemma 4.1] we know that $B^{*}$ is an isomorphism from $M$ onto $V^{o}$. Hence, there exists a unique $u \in M$ such that $B^{*}(u)=\mathcal{F}-A(t, \sigma)$. Thus, $((t, \sigma), u) \in X \times M$ is the unique solution of problem (2.2).

We show now that (i) and (ii) are necessary. Let $\mathcal{G} \in M^{\prime}$ and set $((t, \sigma), u) \in$ $X \times M$ be the unique solution of problem $(2.2)$ with $\mathcal{F} \equiv 0$. Since $B(t, \sigma)=\mathcal{G}$, the range of $B$ is $M^{\prime}$. Thus, using that $X=V \oplus V^{\perp}$ and that $B$ is bounded, we deduce that $B$ is an isomorphism from $V^{\perp}$ onto $M^{\prime}$. Therefore, again by [12: Chapter I/Lemma 4.1] we conclude that condition (i) holds.

On the other hand, given $(\mathcal{F}, \mathcal{G}) \in X^{\prime} \times M^{\prime}$, there exists a unique $((t, \sigma), u) \in$ $X \times M$ solution of problem (2.2). It follows that $(t, \sigma) \in V(\mathcal{G})$ and that $(t, \sigma)$ is a solution of problem (2.3). Let $(\tilde{t}, \tilde{\sigma}) \in V(\mathcal{G})$ be such that $(\tilde{t}, \tilde{\sigma})$ is also a solution of problem (2.3). This implies that $(\mathcal{F}-A(\tilde{t}, \tilde{\sigma})) \in V^{o}$. Now, since (i) holds, $B^{*}$ is an isomorphism from $M$ onto $V^{o}$, and hence there exists a unique $\tilde{u} \in M$ such that $B^{*}(\tilde{u})=(\mathcal{F}-A(\tilde{t}, \tilde{\sigma}))$. It follows that both $((t, \sigma), u)$ and $((\tilde{t}, \tilde{\sigma}), \tilde{u})$ are solutions of problem $(2.2)$, and therefore $((t, \sigma), u)=((\tilde{t}, \tilde{\sigma}), \tilde{u})$. This proves (ii) and completes the proof of the theorem

In the sequel, we analyze problem (2.3) according to the structure of $A$ given by (2.1). From now on, we assume that $B$ satisfies the continuous inf-sup Babuska-Brezzi condition (2.4). Then, by [12: Chapter I/Lemma 4.1], there exists $\left(t_{0}, \sigma_{0}\right) \in X$ such that $B\left(t_{0}, \sigma_{0}\right)=\mathcal{G}$. Thus, problem (2.3) can be replaced by the following problem:

Find $(\tilde{t}, \tilde{\sigma}) \in V$ such that $\Pi A\left((\tilde{t}, \tilde{\sigma})+\left(t_{0}, \sigma_{0}\right)\right)=\Pi(\mathcal{F})$, that is

$$
\left[A\left((\tilde{t}, \tilde{\sigma})+\left(t_{0}, \sigma_{0}\right)\right),(s, \tau)\right]_{X^{\prime} \times X}=[\mathcal{F},(s, \tau)]_{X^{\prime} \times X}
$$

for all $(s, \tau) \in V$.

Now, set $V=\widetilde{X}_{1} \times \widetilde{M}_{1}$ with $\widetilde{X}_{1}$ and $\widetilde{M}_{1}$ subspaces of $X_{1}$ and $M_{1}$, respectively. Further, let

$$
\left.\begin{array}{rl}
P_{1}: \widetilde{X}_{1} \rightarrow X_{1} \\
Q_{1}: \widetilde{M}_{1} \rightarrow M_{1}
\end{array}\right\} \quad \text { and } \quad\left\{\begin{array}{c}
P_{1}^{\prime}: X_{1}^{\prime} \rightarrow \widetilde{X}_{1}^{\prime} \\
Q_{1}^{\prime}: M_{1}^{\prime} \rightarrow \widetilde{M}_{1}^{\prime}
\end{array}\right.
$$

be the canonical injections with respective adjoints. Then, denoting $\mathcal{F}=\left(\mathcal{F}_{1}, \mathcal{G}_{1}\right)$ with $\mathcal{F}_{1} \in X_{1}^{\prime}$ and $\mathcal{G}_{1} \in M_{1}^{\prime}$ and using (2.1), problem (2.5) reduces to the following one: 
Find $(\tilde{t}, \tilde{\sigma}) \in V$ such that

$$
\left.\begin{array}{rl}
P_{1}^{\prime} A_{1}\left(\tilde{t}+t_{0}\right)+P_{1}^{\prime} B_{1}^{*}(\tilde{\sigma}) & =P_{1}^{\prime} \widetilde{\mathcal{F}}_{1} \\
Q_{1}^{\prime} B_{1}(\tilde{t}) & =Q_{1}^{\prime} \widetilde{\mathcal{G}}_{1}
\end{array}\right\}
$$

where $\widetilde{\mathcal{F}}_{1}=\mathcal{F}_{1}-B_{1}^{*}\left(\sigma_{0}\right) \in X_{1}^{\prime}$ and $\widetilde{\mathcal{G}}_{1}=\mathcal{G}_{1}-B_{1}\left(t_{0}\right) \in M_{1}^{\prime}$.

Equivalently, (2.6) reads:

Find $(\tilde{t}, \tilde{\sigma}) \in V=\widetilde{X}_{1} \times \widetilde{M}_{1}$ such that

$$
\left.\begin{array}{rl}
{\left[A_{1}\left(\tilde{t}+t_{0}\right), s\right]_{X_{1}^{\prime} \times X_{1}}+\left[B_{1}^{*}(\tilde{\sigma}), s\right]_{X_{1}^{\prime} \times X_{1}}=\left[\widetilde{\mathcal{F}}_{1}, s\right]_{X_{1}^{\prime} \times X_{1}}} \\
{\left[B_{1}(\tilde{t}), \tau\right]_{M_{1}^{\prime} \times M_{1}}=\left[\widetilde{\mathcal{G}}_{1}, \tau\right]_{M_{1}^{\prime} \times M_{1}}}
\end{array}\right\}
$$

for all $(s, \tau) \in \widetilde{X}_{1} \times \widetilde{M}_{1}$.

Next, we set

$$
\begin{aligned}
V_{1}\left(\widetilde{\mathcal{G}}_{1}\right) & =\left\{s \in \widetilde{X}_{1}: Q_{1}^{\prime} B_{1}(s)=Q_{1}^{\prime} \widetilde{\mathcal{G}}_{1}\right\} \\
& =\left\{s \in \widetilde{X}_{1}:\left[B_{1}(s), \tau\right]_{M_{1}^{\prime} \times M_{1}}=\left[\widetilde{\mathcal{G}}_{1}, \tau\right]_{M_{1}^{\prime} \times M_{1}} \forall \tau \in \widetilde{M}_{1}\right\} \\
V_{1}=V_{1}(O) & =\left\{s \in \widetilde{X}_{1}: Q_{1}^{\prime} B_{1}(s)=0\right\} \\
& =\left\{s \in \widetilde{X}_{1}:\left[B_{1}(s), \tau\right]_{M_{1}^{\prime} \times M_{1}}=0 \forall \tau \in \widetilde{M}_{1}\right\} .
\end{aligned}
$$

Also, we let $\Pi_{1}: X_{1}^{\prime} \rightarrow V_{1}^{\prime}$ be the canonical injection defined by $\Pi_{1}\left(\mathcal{F}_{1}\right)=\left.\mathcal{F}_{1}\right|_{V_{1}}$ for all $\mathcal{F}_{1} \in X_{1}^{\prime}$. Then, we associate to (2.7) the following problem:

Find $\tilde{t} \in V_{1}\left(\widetilde{\mathcal{G}}_{1}\right)$ such that $\Pi_{1} A_{1}\left(\tilde{t}+t_{0}\right)=\Pi_{1}\left(\widetilde{\mathcal{F}}_{1}\right)$, that is

$$
\left[A_{1}\left(\tilde{t}+t_{0}\right), s\right]_{X_{1}^{\prime} \times X_{1}}=\left[\widetilde{\mathcal{F}}_{1}, s\right]_{X_{1}^{\prime} \times X_{1}}
$$

for all $s \in V_{1}$.

Thus, as a consequence of Theorem 2.1, we obtain the following result.

Theorem 2.2. There exists a unique $(\tilde{t}, \tilde{\sigma}) \in V$ solution of problem (2.7) if and only if the following conditions hold:

(i) There exists $\beta_{1}>0$ such that

$$
\sup _{0 \neq s \in \widetilde{X}_{1}} \frac{\left[B_{1}(s), \tau\right]_{M_{1}^{\prime} \times M_{1}}}{\|s\|_{X_{1}}} \geq \beta_{1}\|\tau\|_{M_{1}}
$$

for all $\tau \in \widetilde{M}_{1}$.

(ii) There exists a unique $\tilde{t} \in V_{1}\left(\widetilde{\mathcal{G}}_{1}\right)$ solution of problem $(2.8)$.

In what follows, we assume that (2.9) holds. Then, using again [12: Chapter I/Lemma 4.1] we deduce that there exists $\tilde{t}_{0} \in \widetilde{X}_{1}$ such that $Q_{1}^{\prime} B_{1}\left(\tilde{t}_{0}\right)=Q_{1}^{\prime} \widetilde{\mathcal{G}}_{1}$. Therefore, problem (2.8) can be replaced by the following one: 
Find $\hat{t} \in V_{1}$ such that $\Pi_{1} A_{1}\left(\hat{t}+\tilde{t}_{0}+t_{0}\right)=\Pi_{1}\left(\widetilde{\mathcal{F}}_{1}\right)$, that is

$$
\left[A_{1}\left(\hat{t}+\tilde{t}_{0}+t_{0}\right), s\right]_{X_{1}^{\prime} \times X_{1}}=\left[\widetilde{\mathcal{F}}_{1}, s\right]_{X_{1}^{\prime} \times X_{1}}
$$

for all $s \in V_{1}$.

The unique solvability of problem (2.10) can now be established by using well known results from nonlinear monotone operator theory.

Theorem 2.3. Assume that for any $\tilde{t} \in X_{1}$ the nonlinear operator $\Pi_{1} A_{1}(\cdot+\tilde{t})$ : $V_{1} \rightarrow V_{1}^{\prime}$ is strongly monotone, that is, there exists a constant $\alpha_{1}>0$ independent of $\tilde{t}$ such that

$$
\left[A_{1}\left(\hat{t}_{1}+\tilde{t}\right)-A_{1}\left(\hat{t}_{2}+\tilde{t}\right), \hat{t}_{1}-\hat{t}_{2}\right]_{X_{1}^{\prime} \times X_{1}} \geq \alpha_{1}\left\|\hat{t}_{1}-\hat{t}_{2}\right\|_{X_{1}}^{2}
$$

for all $\hat{t}_{1}, \hat{t}_{2} \in V_{1}$. In addition, assume that $A_{1}: X_{1} \rightarrow X_{1}^{\prime}$ is Lipschitz continuous, that is there exists a constant $\gamma>0$ such that

$$
\sup _{0 \neq s \in X_{1}} \frac{\left|\left[A_{1}\left(t_{1}\right)-A_{1}\left(t_{2}\right), s\right]_{X_{1}^{\prime} \times X_{1}}\right|}{\|s\|_{X_{1}}} \leq \gamma\left\|t_{1}-t_{2}\right\|_{X_{1}}
$$

for all $t_{1}, t_{2} \in X_{1}$. Then, there exists a unique $\hat{t} \in V_{1}$ solution of problem (2.10).

Proof. The proof follows from a usual result in nonlinear functional analysis (see, e.g., [18: Chapter III/Theorem 3.3.23])

Under the assumptions of Theorem 2.3, we remark that the solution $\tilde{t}=\left(\hat{t}+\tilde{t}_{0}\right) \in$ $V_{1}\left(\widetilde{\mathcal{G}}_{1}\right)$ of problem $(2.8)$ is independent of the choice of $\tilde{t}_{0} \in V_{1}\left(\widetilde{\mathcal{G}}_{1}\right)$. In fact, let $\bar{t}_{0} \in V_{1}\left(\widetilde{\mathcal{G}}_{1}\right)$ and let $\bar{t} \in V_{1}$ be the unique solution of

$$
\left[A_{1}\left(\bar{t}+\bar{t}_{0}+t_{0}\right), s\right]_{X_{1}^{\prime} \times X_{1}}=\left[\widetilde{\mathcal{F}}_{1}, s\right]_{X_{1}^{\prime} \times X_{1}}
$$

for all $s \in V_{1}$. Since

$$
\left[A_{1}\left(\bar{t}+\bar{t}_{0}+t_{0}\right), s\right]_{X_{1}^{\prime} \times X_{1}}=\left[A_{1}\left(\left(\bar{t}+\bar{t}_{0}-\tilde{t}_{0}\right)+\tilde{t}_{0}+t_{0}\right), s\right]_{X_{1}^{\prime} \times X_{1}}
$$

we deduce, according to the uniqueness of solution of problem (2.10) with $\tilde{t}_{0}$, that $\bar{t}+\bar{t}_{0}-\tilde{t}_{0}=\hat{t}$, which yields $\hat{t}+\tilde{t}_{0}=\bar{t}+\bar{t}_{0} \in V_{1}\left(\widetilde{\mathcal{G}}_{1}\right)$. Similarly, under the assumptions of Theorems 2.2 and 2.3 , it is easy to prove that the solution $(t, \sigma)=\left((\tilde{t}, \tilde{\sigma})+\left(t_{0}, \sigma_{0}\right)\right) \in$ $V(\mathcal{G})$ of problem $(2.3)$ is independent of the choice of $\left(t_{0}, \sigma_{0}\right) \in V(\mathcal{G})$.

We are now in a position to state the main result concerning the solvability of our original problem (2.2).

Theorem 2.4. With the definitions and notations indicated above, assume the following:

(i) There exists a constant $\beta>0$ such that

$$
\sup _{0 \neq(s, \tau) \in X} \frac{[B(s, \tau), v]_{M^{\prime} \times M}}{\|(s, \tau)\|_{X}} \geq \beta\|v\|_{M}
$$


for all $v \in M$.

(ii) There exists a constant $\beta_{1}>0$ such that

$$
\sup _{0 \neq s \in \widetilde{X}_{1}} \frac{\left[B_{1}(s), \tau\right]_{M_{1}^{\prime} \times M_{1}}}{\|s\|_{X_{1}}} \geq \beta_{1}\|\tau\|_{M_{1}}
$$

for all $\tau \in \widetilde{M}_{1}$.

(iii) The nonlinear operator $A_{1}: X_{1} \rightarrow X_{1}^{\prime}$ is Lipschitz continuous, and for any $\tilde{t} \in X_{1}$, the nonlinear operator $\Pi_{1} A_{1}(\cdot+\tilde{t}): V_{1} \rightarrow V_{1}^{\prime}$ is strongly monotone, as described in Theorem 2.3 .

Then, for each $(\mathcal{F}, \mathcal{G}) \in X^{\prime} \times M^{\prime}$ there exists a unique $((t, \sigma), u) \in X \times M$ solution of problem (2.2).

Proof. The proof follows straightforward from Theorems $2.1-2.3$

\section{The discrete problem}

Let $X_{1, h}, M_{1, h}$ and $M_{h}$ be finite-dimensional subspaces of $X_{1}, M_{1}$ and $M$, respectively, and let $X_{h}=X_{1, h} \times M_{1, h}$ be the corresponding subspace of $X$. In addition, let

$$
\left.\begin{array}{rl}
p_{h}: X_{1, h} & \rightarrow X_{1} \\
q_{h}: M_{1, h} & \rightarrow M_{1} \\
i_{h}=\left(p_{h}, q_{h}\right): X_{h} \rightarrow X \\
j_{h}: M_{h} \rightarrow M
\end{array}\right\} \quad \text { and } \quad\left\{\begin{array}{c}
p_{h}^{\prime}: X_{1}^{\prime} \rightarrow X_{1, h}^{\prime} \\
q_{h}^{\prime}: M_{1}^{\prime} \rightarrow M_{1, h}^{\prime} \\
i_{h}^{\prime}: X^{\prime} \rightarrow X_{h}^{\prime} \\
j_{h}^{\prime}: M^{\prime} \rightarrow M_{h}^{\prime}
\end{array}\right.
$$

be the canonical injections with respetive adjoints. Then, we define the nonlinear operators

$$
\begin{aligned}
A_{1, h} & =p_{h}^{\prime} A_{1}: X_{1} \rightarrow X_{1, h}^{\prime} \\
A_{h} & =i_{h}^{\prime} A: X \rightarrow X_{h}^{\prime}
\end{aligned}
$$

and the linear bounded operators with respective transposes

$$
\left.\begin{array}{c}
B_{1, h}=q_{h}^{\prime} B_{1}: X_{1} \rightarrow M_{1, h}^{\prime} \\
B_{h}=j_{h}^{\prime} B: X \rightarrow M_{h}^{\prime}
\end{array}\right\} \quad \text { and } \quad\left\{\begin{array}{c}
B_{1, h}^{*}=p_{h}^{\prime} B_{1}^{*}: M_{1} \rightarrow X_{1, h}^{\prime} \\
B_{h}^{*}=i_{h}^{\prime} B^{*}: M \rightarrow X_{h}^{\prime} .
\end{array}\right.
$$

Hence, the Galerkin scheme associated with (2.2) reads as follows:

$$
\text { Given } \left.\begin{array}{rl}
(\mathcal{F}, \mathcal{G}) \in X^{\prime} \times M^{\prime}, \text { find }\left(\left(t_{h}, \sigma_{h}\right), u_{h}\right) \in X_{h} \times M_{h} \text { such that } \\
{\left[A_{h}\left(t_{h}, \sigma_{h}\right),\left(s_{h}, \tau_{h}\right)\right]_{h}+\left[B_{h}^{*}\left(u_{h}\right),\left(s_{h}, \tau_{h}\right)\right]_{h}=\left[i_{h}^{\prime} \mathcal{F},\left(s_{h}, \tau_{h}\right)\right]_{h}} \\
{\left[B_{h}\left(t_{h}, \sigma_{h}\right), v_{h}\right]_{h}=\left[j_{h}^{\prime} \mathcal{G}, v_{h}\right]_{h}}
\end{array}\right\}
$$

for all $\left(\left(s_{h}, \tau_{h}\right), v_{h}\right) \in X_{h} \times M_{h}$ where hereafter $[\cdot, \cdot]_{h}$ denotes the duality pairing in the corresponding finite-dimensional subspaces, according to the above definitions of the discrete operators. 
Now, similarly as in Section 2 we set

$$
\begin{aligned}
V_{h}(\mathcal{G}) & =\left\{\left(s_{h}, \tau_{h}\right) \in X_{h}: B_{h}\left(s_{h}, \tau_{h}\right)=j_{h}^{\prime} \mathcal{G}\right\} \\
& =\left\{\left(s_{h}, \tau_{h}\right) \in X_{h}:\left[B_{h}\left(s_{h}, \tau_{h}\right), v_{h}\right]_{h}=\left[\mathcal{G}, v_{h}\right]_{h} \forall v_{h} \in M_{h}\right\} \\
V_{h}=V_{h}(0) & =\left\{\left(s_{h}, \tau_{h}\right) \in X_{h}: B_{h}\left(s_{h}, \tau_{h}\right)=O\right\} \\
& =\left\{\left(s_{h}, \tau_{h}\right) \in X_{h}:\left[B_{h}\left(s_{h}, \tau_{h}\right), v_{h}\right]_{h}=0 \forall v_{h} \in M_{h}\right\} .
\end{aligned}
$$

Thus, with (3.1) we associate the following problem:

Find $\left(t_{h}, \sigma_{h}\right) \in V_{h}(\mathcal{G})$ such that

$$
\left[A_{h}\left(t_{h}, \sigma_{h}\right),\left(s_{h}, \tau_{h}\right)\right]_{h}=\left[i_{h}^{\prime} \mathcal{F},\left(s_{h}, \tau_{h}\right)\right]_{h}
$$

for all $\left(s_{h}, \tau_{h}\right) \in V_{h}$.

It is clear that if $\left(\left(t_{h}, \sigma_{h}\right), u_{h}\right) \in X_{h} \times M_{h}$ is a solution of problem (3.1), then $\left(t_{h}, \sigma_{h}\right) \in V_{h}(\mathcal{G})$ and $\left(t_{h}, \sigma_{h}\right)$ is a solution of problem $(3.2)$ since for all $\left(s_{h}, \tau_{h}\right) \in V_{h}$ one has

$$
\left[B_{h}^{*}\left(u_{h}\right),\left(s_{h}, \tau_{h}\right)\right]_{h}=\left[B_{h}\left(s_{h}, \tau_{h}\right), u_{h}\right]_{h}=0 .
$$

Conversely, if $\left(t_{h}, \sigma_{h}\right) \in V_{h}(\mathcal{G})$ is a solution of problem (3.2), and if $B_{h}$ satisfies the discrete inf-sup Babuska-Brezzi condition (see (3.3) below), then there exists $u_{h} \in M_{h}$ such that $\left(\left(t_{h}, \sigma_{h}\right), u_{h}\right) \in X_{h} \times M_{h}$ is a solution of problem (3.1).

In other words, the previous comments indicate that the discrete analogue of Theorem 2.1 also holds. Since its proof is almost identical to the continuous one from Section 2, we omit further details. Therefore, to continue our analysis we assume that there exists $\beta^{*}>0$ independent of the subspaces involved such that

$$
\sup _{0 \neq\left(s_{h}, \tau_{h}\right) \in X_{h}} \frac{\left[B_{h}\left(s_{h}, \tau_{h}\right), v_{h}\right]_{h}}{\left\|\left(s_{h}, \tau_{h}\right)\right\|_{X}} \geq \beta^{*}\left\|v_{h}\right\|_{M}
$$

for all $v_{h} \in M_{h}$. This inequality states that $B_{h}$ and $B_{h}^{*}$ are isomorphisms from $V_{h}^{\perp} \cap X_{h}$ onto $M_{h}^{\prime}$ and from $M_{h}$ onto $V_{h}^{o} \cap X_{h}^{\prime}$, respectively. In particular, there exists a unique $\left(t_{0, h}, \sigma_{0, h}\right) \in V_{h}^{\perp} \cap X_{h}$ such that $B_{h}\left(t_{0, h}, \sigma_{0, h}\right)=j_{h}^{\prime} \mathcal{G}$, which implies that $V_{h}(\mathcal{G})$ is non-empty. As a consequence of this fact, problem (3.2) can be replaced by the following one:

Find $\left(\tilde{t}_{h}, \tilde{\sigma}_{h}\right) \in V_{h}$ such that

$$
\left[A_{h}\left(\left(\tilde{t}_{h}, \tilde{\sigma}_{h}\right)+\left(t_{0, h}, \sigma_{0, h}\right)\right),\left(\tilde{s}_{h}, \tilde{\tau}_{h}\right)\right]_{h}=\left[i_{h}^{\prime} \mathcal{F},\left(\tilde{s}_{h}, \tilde{\tau}_{h}\right)\right]_{h}
$$

for all $\left(\tilde{s}_{h}, \tilde{\tau}_{h}\right) \in V_{h}$.

In this way, given a unique solution $\left(\tilde{t}_{h}, \tilde{\sigma}_{h}\right) \in V_{h}$ of problem (3.4), the pair $\left(t_{h}, \sigma_{h}\right)=\left(\tilde{t}_{h}+t_{0, h}, \tilde{\sigma}_{h}+\sigma_{0, h}\right) \in V_{h}(\mathcal{G})$ becomes the unique solution of problem $(3.2)$. 
Now, in order to study the solvability of problem (3.4), we note first that we can set

$$
V_{h}=\widetilde{X}_{1, h} \times \widetilde{M}_{1, h}
$$

where $\widetilde{X}_{1, h}$ and $\widetilde{M}_{1, h}$ are subspaces of $X_{1, h}$ and $M_{1, h}$, respectively. Also, we recall that $\mathcal{F}=\left(\mathcal{F}_{1}, \mathcal{G}_{1}\right)$ with $\mathcal{F}_{1} \in X_{1}^{\prime}$ and $\mathcal{G}_{1} \in M_{1}^{\prime}$. Then, problem (3.4) can be rewritten in the following manner:

Find $\left(\tilde{t}_{h}, \tilde{\sigma}_{h}\right) \in V_{h}$ such that

$$
\left.\begin{array}{rl}
{\left[A_{1, h}\left(\tilde{t}_{h}+t_{0, h}\right), \tilde{s}_{h}\right]_{h}+\left[B_{1, h}^{*}\left(\tilde{\sigma}_{h}\right), \tilde{s}_{h}\right]_{h}} & =\left[\tilde{\mathcal{F}}_{1, h}, \tilde{s}_{h}\right]_{h} \\
{\left[B_{1, h}\left(\tilde{t}_{h}\right), \tilde{\tau}_{h}\right]_{h}} & =\left[\widetilde{\mathcal{G}}_{1, h}, \tilde{\tau}_{h}\right]_{h}
\end{array}\right\}
$$

for all $\left(\tilde{s}_{h}, \tilde{\tau}_{h}\right) \in \widetilde{X}_{1, h} \times \widetilde{M}_{1, h}$, where

$$
\begin{aligned}
& \widetilde{\mathcal{F}}_{1, h}=p_{h}^{\prime} \mathcal{F}_{1}-B_{1, h}^{*}\left(\sigma_{0, h}\right) \in X_{1, h}^{\prime} \\
& \widetilde{\mathcal{G}}_{1, h}=q_{h}^{\prime} \mathcal{G}_{1}-B_{1, h}\left(t_{0, h}\right) \in M_{1, h}^{\prime} .
\end{aligned}
$$

We observe that (3.5) is a non-conforming Galerkin approximation scheme for (2.7) since $\left(t_{h}, \sigma_{0, h}\right)$ does not necessarily coincide with $\left(t_{0}, \sigma_{0}\right)$ and moreover, $V_{h}$ is not necessarily a subspace of $V$. This last fact will again be reflected in the derivation of the error estimate in Section 4.

Next, we let $P_{1, h}: \widetilde{X}_{1, h} \rightarrow X_{1, h}$ and $Q_{1, h}: \widetilde{M}_{1, h} \rightarrow M_{1, h}$ be the canonical injections with adjoints $P_{1, h}^{\prime}: X_{1, h}^{\prime} \rightarrow \widetilde{X}_{1, h}^{\prime}$ and $Q_{1, h}^{\prime}: M_{1, h}^{\prime} \rightarrow \widetilde{M}_{1, h}^{\prime}$, and define

$$
\begin{aligned}
V_{1, h}\left(\widetilde{\mathcal{G}}_{1, h}^{\prime}\right) & =\left\{\tilde{s}_{h} \in \tilde{X}_{1, h}: Q_{1, h}^{\prime} B_{1, h}\left(\tilde{s}_{h}\right)=Q_{1, h}^{\prime} \widetilde{\mathcal{G}}_{1, h}\right\} \\
& =\left\{\tilde{s}_{h} \in \widetilde{X}_{1, h}:\left[B_{1, h}\left(\tilde{s}_{h}\right), \tilde{\tau}_{h}\right]_{h}=\left[\widetilde{\mathcal{G}}_{1, h}, \tilde{\tau}_{h}\right]_{h} \forall \tilde{\tau}_{h} \in \widetilde{M}_{1, h}\right\} \\
V_{1, h}=V_{1, h}(0) & =\left\{\tilde{s}_{h} \in \widetilde{X}_{1, h}: Q_{1, h}^{\prime} B_{1, h}\left(\tilde{s}_{h}\right)=0\right\} \\
& =\left\{\tilde{s}_{h} \in \tilde{X}_{1, h}:\left[B_{1, h}\left(\tilde{s}_{h}\right), \tilde{\tau}_{h}\right]_{h}=0 \forall \tilde{\tau}_{h} \in \widetilde{M}_{1, h}\right\} .
\end{aligned}
$$

Also, let $\Pi_{1, h}: X_{1, h}^{\prime} \rightarrow V_{1, h}^{\prime}$ be the canonical injection defined by $\Pi_{1, h}\left(\mathcal{F}_{1, h}\right)=$ $\left.\mathcal{F}_{1, h}\right|_{V_{1, h}}$ for all $\mathcal{F}_{1, h} \in X_{1, h}^{\prime}$. Then, we associate to (3.5) the following problem:

Find $\tilde{t}_{h} \in V_{1, h}\left(\widetilde{\mathcal{G}}_{1, h}\right)$ such that $\Pi_{1, h} A_{1, h}\left(\tilde{t}_{h}+t_{0, h}\right)=\Pi_{1, h}\left(\widetilde{\mathcal{F}}_{1, h}\right)$, that is

$$
\left[A_{1, h}\left(\tilde{t}_{h}+t_{0, h}\right), \tilde{s}_{h}\right]_{h}=\left[\widetilde{\mathcal{F}}_{1, h}, \tilde{s}_{h}\right]_{h}
$$

for all $\tilde{s}_{h} \in V_{1, h}$.

Similarly as before, we remark now that the discrete analogue of Theorem 2.2 also holds. Consequently, for the rest of the present analysis, we suppose that there exists a constant $\beta_{1}^{*}>0$ independent of the subspaces involved such that

$$
\sup _{0 \neq \tilde{s}_{h} \in \widetilde{X}_{1, h}} \frac{\left[B_{1, h}\left(\tilde{s}_{h}\right), \tilde{\tau}_{h}\right]_{h}}{\left\|\tilde{s}_{h}\right\|_{X_{1}}} \geq \beta_{1}^{*}\left\|\tilde{\tau}_{h}\right\|_{M_{1}}
$$


for all $\tilde{\tau}_{h} \in \widetilde{M}_{1, h}$.

The above discrete inf-sup Babuska-Brezzi condition establishes that $Q_{1, h}^{\prime} B_{1, h}$ and $B_{1, h}^{*} Q_{1, h}$ are isomorphisms from $V_{1, h}^{\perp} \cap \widetilde{X}_{1, h}$ onto $\widetilde{M}_{1, h}^{\prime}$ and from $\widetilde{M}_{1, h}$ onto $V_{1, h}^{o} \cap X_{1, h}^{\prime}$, respectively. In particular, there exists a unique $\tilde{t}_{0, h} \in V_{1, h}^{\perp} \cap \widetilde{X}_{1, h}$ such that $Q_{1, h}^{\prime} B_{1, h}\left(\tilde{t}_{0, h}\right)=Q_{1, h}^{\prime} \widetilde{\mathcal{G}}_{1, h}$, which proves that $V_{1, h}\left(\widetilde{\mathcal{G}}_{1, h}\right)$ is non-empty. Therefore, instead of (3.10) we set the following equivalent problem:

Find $\hat{t}_{h} \in V_{1, h}$ such that

$$
\left[A_{1, h}\left(\hat{t}_{h}+\tilde{t}_{0, h}+t_{0, h}\right), \tilde{s}_{h}\right]_{h}=\left[\widetilde{\mathcal{F}}_{1, h}, \tilde{s}_{h}\right]_{h}
$$

for all $\tilde{s}_{h} \in V_{1, h}$.

This means that given a unique solution $\hat{t}_{h} \in V_{1, h}$ of problem (3.12), the vector $\tilde{t}_{h}=\left(\hat{t}_{h}+\tilde{t}_{0, h}\right) \in V_{1, h}\left(\widetilde{\mathcal{G}}_{1, h}\right)$ becomes the unique solution of problem (3.10).

The unique solvability of problem (3.12), which is the discrete analogue of Theorem 2.3, is stated as follows.

Theorem 3.1. Assume that for any $\tilde{t} \in X_{1, h}$ the nonlinear operator

$$
\Pi_{1, h} A_{1, h}(\cdot+\tilde{t}): V_{1, h} \rightarrow V_{1, h}^{\prime}
$$

is strongly monotone, that is there exists a constant $\alpha_{h}>0$ independent of $\tilde{t}$ such that

$$
\left[A_{1, h}\left(\hat{t}_{1, h}+\tilde{t}\right)-A_{1, h}\left(\hat{t}_{2, h}+\tilde{t}\right), \hat{t}_{1, h}-\hat{t}_{2, h}\right]_{h} \geq \alpha_{h}\left\|\hat{t}_{1, h}-\hat{t}_{2, h}\right\|_{X_{1}}^{2}
$$

for all $\hat{t}_{1, h}, \hat{t}_{2, h} \in V_{1, h}$. In addition, assume that $A_{1, h}: X_{1} \rightarrow X_{1, h}^{\prime}$ is Lipschitz continuous, that is there exists a constant $\gamma_{h}>0$ such that

$$
\sup _{0 \neq s_{h} \in X_{1, h}} \frac{\left|\left[A_{1, h}\left(t_{1}\right)-A_{1, h}\left(t_{2}\right), s_{h}\right]_{h}\right|}{\left\|s_{h}\right\|_{X_{1}}} \leq \gamma_{h}\left\|t_{1}-t_{2}\right\|_{X_{1}}
$$

for all $t_{1}, t_{2} \in X_{1}$. Then, there exists a unique $\hat{t}_{h} \in V_{1, h}$ solution of problem (3.12).

Proof. The proof follows from [18: Chapter III/Theorem 3.3.23]

Clearly, the Lipschitz continuity of $A_{1}$ yields the same property of $A_{1, h}$, with the same Lipschitz constant $\gamma$, independent of $h$, given in Theorem 2.3.

At this point, we remark as for the continuous case that the solution

$$
\tilde{t}_{h}=\left(\hat{t}_{h}+\tilde{t}_{0, h}\right) \in V_{1, h}\left(\widetilde{\mathcal{G}}_{1, h}\right)
$$

of problem (3.10) is independent of the choice of $\tilde{t}_{0, h} \in V_{1, h}\left(\widetilde{\mathcal{G}}_{1, h}\right)$. Also, the solution

$$
\left(t_{h}, \sigma_{h}\right)=\left(\tilde{t}_{h}+t_{0, h}, \tilde{\sigma}_{h}+\sigma_{0, h}\right) \in V_{h}(\mathcal{G})
$$

of problem (3.2) is independent of the choice of $\left(t_{0, h}, \sigma_{0, h}\right) \in V_{h}(\mathcal{G})$.

According to our previous analysis, we have already proved the following theorem concerning the solvability of the Galerkin scheme (3.1). 
Theorem 3.2. With the definitions and notations indicated above, assume the following:

(i) There exists a constant $\beta^{*}>0$ independent of the subspaces involved such that

$$
\sup _{0 \neq\left(s_{h}, \tau_{h}\right) \in X_{h}} \frac{\left[B_{h}\left(s_{h}, \tau_{h}\right), v_{h}\right]_{h}}{\left\|\left(s_{h}, \tau_{h}\right)\right\|_{X}} \geq \beta^{*}\left\|v_{h}\right\|_{M}
$$

for all $v_{h} \in M_{h}$.

(ii) There exists a constant $\beta_{1}^{*}>0$ independent of the subspaces involved such that

$$
\sup _{0 \neq \tilde{s}_{h} \in \widetilde{X}_{1, h}} \frac{\left[B_{1, h}\left(\tilde{s}_{h}\right), \tilde{\tau}_{h}\right]_{h}}{\left\|\tilde{s}_{h}\right\|_{X_{1}}} \geq \beta_{1}^{*}\left\|\tilde{\tau}_{h}\right\|_{M_{1}}
$$

for all $\tilde{\tau}_{h} \in \widetilde{M}_{1, h}$.

(iii) The nonlinear operator $A_{1, h}: X_{1} \rightarrow X_{1, h}^{\prime}$ is Lipschitz continuous, and for any $\tilde{t} \in X_{1, h}$ the nonlinear operator $\Pi_{1, h} A_{1, h}(\cdot+\tilde{t}): V_{1, h} \rightarrow V_{1, h}^{\prime}$ is strongly monotone, as described in Theorem 3.1.

Then, for each $(\mathcal{F}, \mathcal{G}) \in X^{\prime} \times M^{\prime}$ there exists a unique $\left(\left(t_{h}, \sigma_{h}\right), u_{h}\right) \in X_{h} \times M_{h}$ solution of problem (3.1).

It remains now to study the error associated with the Galerkin scheme (3.1). This is, precisely, the purpose of the next section.

\section{The error analysis}

In what follows we assume that the index $h$, which identifies the finite-dimensional subspaces, is taken in a numerable family $I=\left\{h_{n}\right\}_{n \in \mathbb{N}}$ such that $h_{j} \geq h_{j+1}>0$ for all $j \in \mathbb{N}$. Throughout this section, we suppose that all the hypotheses of both Theorems 2.4 and 3.2 are satisfied. In addition we suppose that, for all $\tilde{t} \in X_{1, h}$, the family of nonlinear operators $\left\{\Pi_{1, h} A_{1, h}(\cdot+\tilde{t})\right\}_{h \in I}$ is uniformly strongly monotone. This means that there exists $\alpha>0$ independent of $\tilde{t}$ such that

$$
\alpha_{h} \geq \alpha \quad(h \in I)
$$

where $\alpha_{h}$ is the constant indicated in Theorem 3.1.

Hence, our main goal is to demonstrate the following theorem.

Theorem 4.1. Let $((t, \sigma), u) \in X \times M$ and $\left(\left(t_{h}, \sigma_{h}\right), u_{h}\right) \in X_{h} \times M_{h}$ be the unique solutions of problems (2.2) and (3.1), respectively. Then there exists a constant $C>0$ 
depending on $\alpha, \gamma,\left\|B_{1}\right\|, \beta_{1}^{*},\|B\|$ and $\beta^{*}$ only such that the Strang-type error estimate

$$
\begin{aligned}
\left\|((t, \sigma), u)-\left(\left(t_{h}, \sigma_{h}\right), u_{h}\right)\right\| \\
\leq C\left\{\inf _{\left(\left(s_{h}, \tau_{h}\right) v_{h}\right) \in X_{h} \times M_{h}}\left\|((t, \sigma), u)-\left(\left(s_{h}, \tau_{h}\right), v_{h}\right)\right\|\right. \\
+\sup _{0 \neq \tilde{s}_{h} \in \widetilde{X}_{1, h}} \frac{\left[\mathcal{F}_{1}-A_{1}(t)-B_{1}^{*}(\sigma), \tilde{s}_{h}\right]}{\left\|\tilde{s}_{h}\right\|} \\
\left.+\sup _{0 \neq \tilde{\tau}_{h} \in \widetilde{M}_{1, h}} \frac{\left[\mathcal{G}_{1}-B_{1}(t), \tilde{\tau}_{h}\right]}{\left\|\tilde{\tau}_{h}\right\|}\right\}
\end{aligned}
$$

holds for all $h \in I$.

It is important to observe from (2.7) that if $\tilde{X}_{1, h} \subseteq \tilde{X}_{1}$, then

$$
\sup _{\tilde{s}_{h} \in \widetilde{X}_{1, h}} \frac{\left[\mathcal{F}_{1}-A_{1}(t)-B_{1}^{*}(\sigma), \tilde{s}_{h}\right]}{\left\|\tilde{s}_{h}\right\|}=0 .
$$

Similarly, if $\widetilde{M}_{1, h} \subseteq \widetilde{M}_{1}$, then

$$
\sup _{0 \neq \tilde{\tau}_{h} \in \widetilde{M}_{1, h}} \frac{\left[\mathcal{G}_{1}-B_{1}(t), \tilde{\tau}_{h}\right]}{\left\|\tilde{\tau}_{h}\right\|}=0 .
$$

It follows that if $V_{h} \subseteq V$, then (4.2) becomes the usual Cea estimate for the Galerkin error. In other words, the second and third terms on the right-hand side of (4.2) constitute the consistency error for the case in which $V_{h}$ is not a subspace of $V$.

Hereafter, for simplicity we omit the subscripts for the norms and for the duality pairings $[\cdot, \cdot]$ since the corresponding meanings will always be clear.

In order to prove Theorem 4.1 we need several previous results. To this end, we recall that

$$
(t, \sigma)=\left(\tilde{t}+t_{0}, \tilde{\sigma}+\sigma_{0}\right) \quad \text { where }\left(t_{0}, \sigma_{0}\right) \in V(\mathcal{G}),(\tilde{t}, \tilde{\sigma}) \in V
$$

is the unique solution of problem (2.7). Also,

$$
\left(t_{h}, \sigma_{h}\right)=\left(\tilde{t}_{h}+t_{0, h}, \tilde{\sigma}_{h}+\sigma_{0, h}\right) \quad \text { where }\left(t_{0, h}, \sigma_{0, h}\right) \in V_{h}(\mathcal{G}),\left(\tilde{t}_{h}, \tilde{\sigma}_{h}\right) \in V_{h}
$$

is the unique solution of problem (3.5). Further,

$$
\tilde{t}_{h}=\hat{t}_{h}+\tilde{t}_{0, h} \quad \text { where } \tilde{t}_{0, h} \in V_{1, h}\left(\widetilde{\mathcal{G}}_{1, h}\right), \hat{t}_{h} \in V_{1, h}
$$

is the unique solution of problem (3.12). 
Lemma 4.4. The estimate

$$
\begin{aligned}
\left\|t-t_{h}\right\| \leq & \frac{1}{\alpha} \sup _{0 \neq \tilde{s}_{h} \in \widetilde{X}_{1, h}} \frac{\left[\mathcal{F}_{1}-A_{1}(t)-B_{1}^{*}(\sigma), \tilde{s}_{h}\right]}{\left\|\tilde{s}_{h}\right\|} \\
& +\left(1+\frac{\gamma}{\alpha}\right) \inf _{s_{h} \in V_{1, h}\left(\mathcal{G}_{1}\right)}\left\|t-s_{h}\right\| \\
& +\frac{\left\|B_{1}\right\|}{\alpha} \inf _{\tilde{\tau}_{h} \in \widetilde{M}_{1, h}}\left\|\sigma-\left(\tilde{\tau}_{h}+\sigma_{0, h}\right)\right\|
\end{aligned}
$$

holds for all $h \in I$ where

$$
V_{1, h}\left(\mathcal{G}_{1}\right)=\left\{s_{h} \in X_{1, h}:\left[B_{1}\left(s_{h}\right), \tilde{\tau}_{h}\right]=\left[\mathcal{G}_{1}, \tilde{\tau}_{h}\right] \forall \tilde{\tau}_{h} \in \widetilde{M}_{1, h}\right\} .
$$

Proof. First, by using the triangle inequality, we get

$$
\left\|t-t_{h}\right\|=\left\|t-\left(\tilde{t}_{h}+t_{0, h}\right)\right\| \leq\left\|t-\left(\tilde{r}_{h}+t_{0, h}\right)\right\|+\left\|\tilde{t}_{h}-\tilde{r}_{h}\right\|
$$

for all $\tilde{r}_{h} \in V_{1, h}\left(\widetilde{\mathcal{G}}_{1, h}\right)$. Moreover, since $\left(\tilde{r}_{h}-\tilde{t}_{0, h}\right) \in V_{1, h}$ we can write

$$
\begin{aligned}
\left(\tilde{t}_{h}-\tilde{r}_{h}\right) & =\left(\tilde{t}_{h}+t_{0, h}\right)-\left(\tilde{r}_{h}+t_{0, h}\right) \\
& =\left(\hat{t}_{h}+\tilde{t}_{0, h}+t_{0, h}\right)-\left(\hat{r}_{h}+\tilde{t}_{0, h}+t_{0, h}\right) \\
& =\left(\hat{t}_{h}-\hat{r}_{h}\right)
\end{aligned}
$$

with $\hat{r}_{h}=\tilde{r}_{h}-\tilde{t}_{0, h}$. In this way, by employing the uniform strong monotonicity of $\Pi_{1, h} A_{1, h}\left(\cdot+\tilde{t}_{0, h}+t_{0, h}\right)$ (cf. Theorem 3.1 and (4.1)) we deduce

$$
\alpha\left\|\tilde{t}_{h}-\tilde{r}_{h}\right\|^{2} \leq\left[A_{1, h}\left(\tilde{t}_{h}+t_{0, h}\right), \tilde{t}_{h}-\tilde{r}_{h}\right]-\left[A_{1, h}\left(\tilde{r}_{h}+t_{0, h}\right), \tilde{t}_{h}-\tilde{r}_{h}\right] .
$$

Now, since $\left(\tilde{t}_{h}-\tilde{r}_{h}\right) \in V_{1, h}$, we can apply (3.10) and use the definition of $\widetilde{\mathcal{F}}_{1, h}$ (cf. (3.6)) to obtain

$$
\begin{aligned}
& {\left[A_{1, h}\left(\tilde{t}_{h}+t_{0, h}\right), \tilde{t}_{h}-\tilde{r}_{h}\right]} \\
& \quad=\left[\widetilde{\mathcal{F}}_{1, h}, \tilde{t}_{h}-\tilde{r}_{h}\right] \\
& \quad=\left[p_{h}^{\prime} \mathcal{F}_{1}, \tilde{t}_{h}-\tilde{r}_{h}\right]-\left[B_{1, h}^{*}\left(\sigma_{0, h}\right), \tilde{t}_{h}-\tilde{r}_{h}\right] \\
& \quad=\left[\mathcal{F}_{1}, \tilde{t}_{h}-\tilde{r}_{h}\right]-\left[B_{1}\left(\tilde{t}_{h}-\tilde{r}_{h}\right), \sigma_{0, h}\right] .
\end{aligned}
$$

Thus, replacing this back into (4.6) and adding and substracting appropriate terms we get

$$
\begin{aligned}
\alpha\left\|\tilde{t}_{h}-\tilde{r}_{h}\right\|^{2} \leq & {\left[\mathcal{F}_{1}, \tilde{t}_{h}-\tilde{r}_{h}\right]-\left[B_{1}\left(\tilde{t}_{h}-\tilde{r}_{h}\right), \sigma_{0, h}\right] } \\
& -\left[A_{1}\left(\tilde{r}_{h}+t_{0, h}\right), \tilde{t}_{h}-\tilde{r}_{h}\right] \\
& +\left[A_{1}(t), \tilde{t}_{h}-\tilde{r}_{h}\right]+\left[B_{1}\left(\tilde{t}_{h}-\tilde{r}_{h}\right), \sigma\right] \\
& -\left[A_{1}(t), \tilde{t}_{h}-\tilde{r}_{h}\right]-\left[B_{1}\left(\tilde{t}_{h}-\tilde{r}_{h}\right), \sigma\right] \\
= & {\left[\mathcal{F}_{1}-A_{1}(t)-B_{1}^{*}(\sigma), \tilde{t}_{h}-\tilde{r}_{h}\right] } \\
& +\left[A_{1}(t)-A_{1}\left(\tilde{r}_{h}+t_{0, h}\right), \tilde{t}_{h}-\tilde{r}_{h}\right] \\
& +\left[B_{1}\left(\tilde{t}_{h}-\tilde{r}_{h}\right), \sigma-\sigma_{0, h}\right] .
\end{aligned}
$$


But, using again that $\left(\tilde{t}_{h}-\tilde{r}_{h}\right) \in V_{1, h}$ which means that $\left[B_{1}\left(\tilde{t}_{h}-\tilde{r}_{h}\right), \tilde{\tau}_{h}\right]=0$ for all $\tilde{\tau}_{h} \in \widetilde{M}_{1, h}$, the last term in (4.8) can be replaced by

$$
\left[B_{1}\left(\tilde{t}_{h}-\tilde{r}_{h}\right), \sigma-\left(\tilde{\tau}_{h}+\sigma_{0, h}\right)\right] .
$$

Therefore, dividing inequality (4.8) by $\alpha\left\|\tilde{t}_{h}-\tilde{r}_{h}\right\|$, using that $V_{1, h} \subseteq \tilde{X}_{1, h}$, applying the Lipschitz continuity of $A_{1}$ and the boundedness of $B_{1}$, we deduce that

$$
\begin{aligned}
\left\|\tilde{t}_{h}-\tilde{r}_{h}\right\| \leq & \frac{1}{\alpha} \sup _{0 \neq \tilde{s}_{h} \in \widetilde{X}_{1, h}} \frac{\left[\mathcal{F}_{1}-A_{1}(t)-B_{1}^{*}(\sigma), \tilde{s}_{h}\right]}{\left\|\tilde{s}_{h}\right\|} \\
& +\frac{\gamma}{\alpha}\left\|t-\left(\tilde{r}_{h}+t_{0, h}\right)\right\| \\
& +\frac{\left\|B_{1}\right\|}{\alpha}\left\|\sigma-\left(\tilde{\tau}_{h}+\sigma_{0, h}\right)\right\|
\end{aligned}
$$

for all $\tilde{r}_{h} \in V_{1, h}\left(\widetilde{\mathcal{G}}_{1, h}\right)$ and all $\tilde{\tau}_{h} \in \widetilde{M}_{1, h}$.

Now, we observe from the definition of $V_{1, h}\left(\widetilde{\mathcal{G}}_{1, h}\right)$ (cf. $\left.(3.7)-(3.8)\right)$ that $\tilde{r}_{h} \in$ $V_{1, h}\left(\widetilde{\mathcal{G}}_{1, h}\right)$ if and only if $\left(\tilde{r}_{h}+t_{0, h}\right) \in V_{1, h}\left(\mathcal{G}_{1}\right)$ (cf. (4.4)). Hence, the second term on the right-hand side of (4.9) and the first term on the right-hand side of (4.5) can be replaced by $\frac{\gamma}{\alpha}\left\|t-s_{h}\right\|$ and $\left\|t-s_{h}\right\|$, respectively, for all $s_{h} \in V_{1, h}\left(\mathcal{G}_{1}\right)$. Consequently, the previous remark together with (4.5) and (4.9) lead the required estimate (4.3), thus completing the proof of the lemma

In order to improve the estimate given by Lemma 4.1, we deduce next a suitable upper bound for the second term on the right-hand side of (4.3).

Lemma 4.2. The estimate

$$
\begin{aligned}
& \inf _{s_{h} \in V_{1, h}\left(\mathcal{G}_{1}\right)}\left\|t-s_{h}\right\| \leq\left(1+\frac{\left\|B_{1}\right\|}{\beta_{1}^{*}}\right) \inf _{\tilde{s}_{h} \in \widetilde{X}_{1, h}}\left\|t-\left(\tilde{s}_{h}+t_{0, h}\right)\right\| \\
& +\frac{1}{\beta_{1}^{*}} \sup _{0 \neq \tilde{\tau}_{h} \in \widetilde{M}_{1, h}} \frac{\left[\mathcal{G}_{1}-B_{1}(t), \tilde{\tau}_{h}\right]}{\left\|\tilde{\tau}_{h}\right\|}
\end{aligned}
$$

holds for all $h \in I$.

Proof. Given $\tilde{s}_{h} \in \widetilde{X}_{1, h}$, we have that $Q_{1, h}^{\prime} B_{1, h}\left(t-\left(\tilde{s}_{h}+t_{0, h}\right)\right) \in \widetilde{M}_{1, h}^{\prime}$. Also, it is clear that $Q_{1, h}^{\prime}\left(q_{h}^{\prime} \mathcal{G}_{1}-B_{1, h}(t)\right) \in \widetilde{M}_{1, h}^{\prime}$. In addition, we recall that the discrete inf-sup condition for $B_{1, h}$ (cf. (3.11)) establishes that $Q_{1, h}^{\prime} B_{1, h}$ is an isomorphism from $\widetilde{X}_{1, h} \cap V_{1, h}^{\perp}$ onto $\widetilde{M}_{1, h}^{\prime}$. It follows that there exists a unique $\bar{s}_{h} \in \widetilde{X}_{1, h} \cap V_{1, h}^{\perp}$ such that

$$
\begin{aligned}
Q_{1, h}^{\prime} B_{1, h}\left(\bar{s}_{h}\right) & =Q_{1, h}^{\prime} B_{1, h}\left(t-\left(\tilde{s}_{h}+t_{0, h}\right)\right)+Q_{1, h}^{\prime}\left(q_{h}^{\prime} \mathcal{G}_{1}-B_{1, h}(t)\right) \\
\left\|\bar{s}_{h}\right\| & \leq \frac{1}{\beta_{1}^{*}}\left\|Q_{1, h}^{\prime} B_{1, h}\left(t-\left(\tilde{s}_{h}+t_{0, h}\right)\right)+Q_{1, h}^{\prime}\left(q_{h}^{\prime} \mathcal{G}_{1}-B_{1, h}(t)\right)\right\|
\end{aligned}
$$

which yields

$$
\left\|\bar{s}_{h}\right\| \leq \frac{\left\|B_{1}\right\|}{\beta_{1}^{*}}\left\|t-\left(\tilde{s}_{h}+t_{0, h}\right)\right\|+\frac{1}{\beta_{1}^{*}} \sup _{0 \neq \tilde{\tau}_{h} \in \widetilde{M}_{1, h}} \frac{\left[\mathcal{G}_{1}-B_{1}(t), \tilde{\tau}_{h}\right]}{\left\|\tilde{\tau}_{h}\right\|} .
$$


We now set $\hat{s}_{h}=\left(\bar{s}_{h}+\tilde{s}_{h}+t_{0, h}\right) \in X_{1, h}$. It is easy to see according to (4.11) that

$$
Q_{1, h}^{\prime} B_{1, h}\left(\hat{s}_{h}\right)=Q_{1, h}^{\prime}\left(q_{h}^{\prime} \mathcal{G}_{1}\right), \quad \text { i.e. }\left[B_{1}\left(\hat{s}_{h}\right), \tilde{\tau}_{h}\right]=\left[\mathcal{G}_{1}, \tilde{\tau}_{h}\right] \quad \forall \tilde{\tau}_{h} \in \widetilde{M}_{1, h}
$$

which shows that $\hat{s}_{h} \in V_{1, h}\left(\mathcal{G}_{1}\right)$. Therefore, we can write

$$
\inf _{s_{h} \in V_{1, h}\left(\mathcal{G}_{1}\right)}\left\|t-s_{h}\right\| \leq\left\|t-\hat{s}_{h}\right\|=\left\|t-\left(\tilde{s}_{h}+t_{0, h}\right)-\bar{s}_{h}\right\| \leq\left\|t-\left(\tilde{s}_{h}+t_{0, h}\right)\right\|+\left\|\bar{s}_{h}\right\|
$$

for all $\tilde{s}_{h} \in \tilde{X}_{1, h}$. Thus, this estimate and (4.12) complete the proof of the lemma

We now provide an upper bound for the error $\left\|\sigma-\sigma_{h}\right\|$.

Lemma 4.3. The estimate

$$
\begin{aligned}
\left\|\sigma-\sigma_{h}\right\| \leq & \frac{1}{\beta_{1}^{*}} \sup _{0 \neq \tilde{s}_{h} \in \widetilde{X}_{1, h}} \frac{\left[\mathcal{F}_{1}-A_{1}(t)-B_{1}^{*}(\sigma), \tilde{s}_{h}\right]}{\left\|\tilde{s}_{h}\right\|} \\
& +\frac{\gamma}{\beta_{1}^{*}}\left\|t-t_{h}\right\|+\left(1+\frac{\left\|B_{1}\right\|}{\beta_{1}^{*}}\right) \inf _{\tilde{\tau}_{h} \in \widetilde{M}_{1, h}}\left\|\sigma-\left(\tilde{\tau}_{h}+\sigma_{0, h}\right)\right\|
\end{aligned}
$$

holds for all $h \in I$.

Proof. We first apply the triangle inequality to obtain

$$
\left\|\sigma-\sigma_{h}\right\|=\left\|\sigma-\left(\tilde{\sigma}_{h}+\sigma_{0, h}\right)\right\| \leq\left\|\sigma-\left(\tilde{\tau}_{h}+\sigma_{0, h}\right)\right\|+\left\|\tilde{\sigma}_{h}-\tilde{\tau}_{h}\right\|
$$

for all $\tilde{\tau}_{h} \in \widetilde{M}_{1, h}$. On the other hand, from the first equation in (3.5) and using the definition of $\widetilde{\mathcal{F}}_{1, h}$ (cf. (3.6)) we get

$$
\left[B_{1, h}^{*}\left(\tilde{\sigma}_{h}+\sigma_{0, h}\right), \tilde{s}_{h}\right]=\left[\mathcal{F}_{1}, \tilde{s}_{h}\right]-\left[A_{1, h}\left(\tilde{t}_{h}+t_{0, h}\right), \tilde{s}_{h}\right]
$$

from where

$$
\begin{aligned}
& {\left[B_{1, h}\left(\tilde{s}_{h}\right), \tilde{\sigma}_{h}-\tilde{\tau}_{h}\right]} \\
& \quad=\left[\mathcal{F}_{1}, \tilde{s}_{h}\right]-\left[A_{1, h}\left(\tilde{t}_{h}+t_{0, h}\right), \tilde{s}_{h}\right]-\left[B_{1, h}\left(\tilde{s}_{h}\right), \tilde{\tau}_{h}+\sigma_{0, h}\right]
\end{aligned}
$$

for all $\left(\tilde{s}_{h}, \tilde{\tau}_{h}\right) \in \widetilde{X}_{1, h} \times \widetilde{M}_{1, h}$. Then, adding and substracting appropriate terms in (4.15) we can write

$$
\begin{aligned}
{\left[B_{1, h}\left(\tilde{s}_{h}\right), \tilde{\sigma}_{h}-\tilde{\tau}_{h}\right]=} & {\left[\mathcal{F}_{1}-A_{1}(t)-B_{1}^{*}(\sigma), \tilde{s}_{h}\right] } \\
& +\left[A_{1}(t), \tilde{s}_{h}\right]-\left[A_{1}\left(\tilde{t}_{h}+t_{0, h}\right), \tilde{s}_{h}\right] \\
& +\left[B_{1}\left(\tilde{s}_{h}\right), \sigma-\left(\tilde{\tau}_{h}+\sigma_{0, h}\right)\right]
\end{aligned}
$$

for all $\left(\tilde{s}_{h}, \tilde{\tau}_{h}\right) \in \widetilde{X}_{1, h} \times \widetilde{M}_{1, h}$. Now, since $\left(\tilde{\sigma}_{h}-\tilde{\tau}_{h}\right) \in \widetilde{M}_{1, h}$ for all $\tilde{\tau}_{h} \in \widetilde{M}_{1, h}$, we can apply the discrete inf-sup condition for $B_{1, h}$ (cf. (3.11)) and deduce that

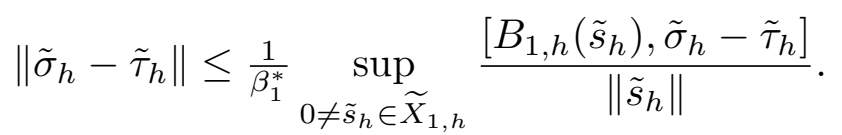

In this way, setting herein (4.16), applying the Lipschitz continuity of $A_{1}$ and the boundedness of $B_{1}$ we conclude that

$$
\begin{aligned}
\left\|\tilde{\sigma}_{h}-\tilde{\tau}_{h}\right\| \leq & \frac{1}{\beta_{1}^{*}} \sup _{0 \neq \tilde{s}_{h} \in \widetilde{X}_{1, h}} \frac{\left[\mathcal{F}_{1}-A_{1}(t)-B_{1}^{*}(\sigma), \tilde{s}_{h}\right]}{\left\|\tilde{s}_{h}\right\|} \\
& +\frac{\gamma}{\beta_{1}^{*}}\left\|t-t_{h}\right\|+\frac{\left\|B_{1}\right\|}{\beta_{1}^{*}}\left\|\sigma-\left(\tilde{\tau}_{h}+\sigma_{0, h}\right)\right\|
\end{aligned}
$$

for all $\tilde{\tau}_{h} \in \widetilde{M}_{1, h}$. Finally, (4.18) and (4.14) finish the proof of the lemma 
As a consequence of the previous lemmata, we can state the following result.

Theorem 4.4. There exists a constant $\tilde{C}>0$ depending only on $\alpha, \gamma,\left\|B_{1}\right\|, \beta_{1}^{*}$, $\|B\|$ and $\beta^{*}$ such that

$$
\begin{aligned}
\left\|(t, \sigma)-\left(t_{h}, \sigma_{h}\right)\right\| \leq & \tilde{C}\left\{\inf _{\left(s_{h}, \tau_{h}\right) \in X_{h}}\left\|(t, \sigma)-\left(s_{h}, \tau_{h}\right)\right\|\right. \\
& +\sup _{0 \neq \tilde{s}_{h} \in \widetilde{X}_{1, h}} \frac{\left[\mathcal{F}_{1}-A_{1}(t)-B_{1}^{*}(\sigma), \tilde{s}_{h}\right]}{\left\|\tilde{s}_{h}\right\|} \\
& \left.+\sup _{0 \neq \tilde{\tau}_{h} \in \widetilde{M}_{1, h}} \frac{\left[\mathcal{G}_{1}-B_{1}(t), \tilde{\tau}_{h}\right]}{\left\|\tilde{\tau}_{h}\right\|}\right\}
\end{aligned}
$$

for all $h \in I$,

Proof. The direct application of Lemmata $4.1-4.3$ yields the estimate

$$
\begin{aligned}
\left\|(t, \sigma)-\left(t_{h}, \sigma_{h}\right)\right\| \leq & C_{1}\left\{\inf _{\left(\tilde{s}_{h}, \tilde{\tau}_{h}\right) \in V_{h}}\left\|(t, \sigma)-\left(\tilde{s}_{h}+t_{0, h}, \tilde{\tau}_{h}+\sigma_{0, h}\right)\right\|\right. \\
& +\sup _{0 \neq \tilde{s}_{h} \in \widetilde{X}_{1, h}} \frac{\left[\mathcal{F}_{1}-A_{1}(t)-B_{1}^{*}(\sigma), \tilde{s}_{h}\right]}{\left\|\tilde{s}_{h}\right\|} \\
& \left.+\sup _{0 \neq \tilde{\tau}_{h} \in \widetilde{M}_{1, h}} \frac{\left[\mathcal{G}_{1}-B_{1}(t), \tilde{\tau}_{h}\right]}{\left\|\tilde{\tau}_{h}\right\|}\right\}
\end{aligned}
$$

where $C_{1}>0$ is a constant depending on $\alpha, \gamma,\left\|B_{1}\right\|$ and $\beta_{1}^{*}$ only. Now, since $\left(t_{0, h}, \sigma_{0, h}\right) \in V_{h}(\mathcal{G})$, we note that $\left(\tilde{s}_{h}, \tilde{\tau}_{h}\right) \in V_{h}$ if and only if $\left(\tilde{s}_{h}+t_{0, h}, \tilde{\tau}_{h}+\sigma_{0, h}\right) \in$ $V_{h}(\mathcal{G})$, and hence

$$
\inf _{\left(\tilde{s}_{h}, \tilde{\tau}_{h}\right) \in V_{h}}\left\|(t, \sigma)-\left(\tilde{s}_{h}+t_{0, h}, \tilde{\tau}_{h}+\sigma_{0, h}\right)\right\|=\inf _{\left(\tilde{s}_{h}, \tilde{\tau}_{h}\right) \in V_{h}(\mathcal{G})}\left\|(t, \sigma)-\left(\tilde{s}_{h}, \tilde{\tau}_{h}\right)\right\| .
$$

Next, we proceed similarly as in the proof of Lemma 4.2. Given $\left(s_{h}, \tau_{h}\right) \in X_{h}$, we have that $B_{h}\left((t, \sigma)-\left(s_{h}, \tau_{h}\right)\right) \in M_{h}^{\prime}$. Also, we recall that the discrete inf-sup condition for $B_{h}$ (cf. (3.3)) states that $B_{h}$ is an isomorphism from $V_{h}^{\perp} \cap X_{h}$ onto $M_{h}^{\prime}$. It follows that there exists a unique $\left(\bar{s}_{h}, \bar{\tau}_{h}\right) \in V_{h}^{\perp} \cap X_{h}$ such that

$$
\begin{aligned}
B_{h}\left(\bar{s}_{h}, \bar{\tau}_{h}\right) & =B_{h}\left((t, \sigma)-\left(s_{h}, \tau_{h}\right)\right) \\
\left\|\left(\bar{s}_{h}, \bar{\tau}_{h}\right)\right\| & \leq \frac{1}{\beta^{*}}\left\|B_{h}\left((t, \sigma)-\left(s_{h}, \tau_{h}\right)\right)\right\| \leq \frac{\|B\|}{\beta^{*}}\left\|(t, \sigma)-\left(s_{h}, \tau_{h}\right)\right\| .
\end{aligned}
$$

Then, we set $\left(\hat{s}_{h}, \hat{\tau}_{h}\right)=\left(\bar{s}_{h}, \bar{\tau}_{h}\right)+\left(s_{h}, \tau_{h}\right)$ and observe from $(4.22)$ that $B_{h}\left(\hat{s}_{h}, \hat{\tau}_{h}\right)=$ $B_{h}(t, \sigma)=j_{h}^{\prime} \mathcal{G}$, which means that $\left(\hat{s}_{h}, \hat{\tau}_{h}\right) \in V_{h}(\mathcal{G})$. Consequently, we deduce that

$$
\begin{aligned}
\inf _{\left(\tilde{s}_{h}, \tilde{\tau}_{h}\right) \in V_{h}(\mathcal{G})}\left\|(t, \sigma)-\left(\tilde{s}_{h}, \tilde{\tau}_{h}\right)\right\| & \leq\left\|(t, \sigma)-\left(\hat{s}_{h}, \hat{\tau}_{h}\right)\right\| \\
& \leq\left\|(t, \sigma)-\left(s_{h}, \tau_{h}\right)\right\|+\left\|\left(\bar{s}_{h}, \bar{\tau}_{h}\right)\right\|
\end{aligned}
$$

for all $\left(s_{h}, \tau_{h}\right) \in X_{h}$. Thus, (4.24) and (4.23) yield

$$
\inf _{\left(s_{h}, \tau_{h}\right) \in V_{h}(\mathcal{G})}\left\|(t, \sigma)-\left(\tilde{s}_{h}, \tilde{\tau}_{h}\right)\right\| \leq\left(1+\frac{\|B\|}{\beta^{*}}\right) \inf _{\left(s_{h}, \tau_{h}\right) \in X_{h}}\left\|(t, \sigma)-\left(s_{h}, \tau_{h}\right)\right\|
$$

which, together with (4.20) and (4.21), completes the proof of the theorem 
The corresponding upper bound for the error $\left\|u-u_{h}\right\|$ is provided next in the usual way.

Theorem 4.5. There exists a constant $\bar{C}>0$ depending on $\gamma,\left\|B_{1}\right\|,\|B\|$ and $\beta^{*}$ only such that

$$
\left\|u-u_{h}\right\| \leq \bar{C}\left\{\left\|(t, \sigma)-\left(t_{h}, \sigma_{h}\right)\right\|+\inf _{v_{h} \in M_{h}}\left\|u-v_{h}\right\|\right\}
$$

for all $h \in I$.

Proof. We first note, by the triangle inequality, that

$$
\left\|u-u_{h}\right\| \leq\left\|u-v_{h}\right\|+\left\|u_{h}-v_{h}\right\|
$$

for all $v_{h} \in M_{h}$. Now, from the first equations in (3.1) and (2.2) we get

$$
\begin{aligned}
& {\left[B_{h}^{*}\left(u_{h}\right),\left(s_{h}, \tau_{h}\right)\right]} \\
& \quad=\left[\mathcal{F},\left(s_{h}, \tau_{h}\right)\right]-\left[A_{h}\left(t_{h}, \sigma_{h}\right),\left(s_{h}, \tau_{h}\right)\right] \\
& \quad=\left[A(t, \sigma),\left(s_{h}, \tau_{h}\right)\right]+\left[B\left(s_{h}, \tau_{h}\right), u\right]-\left[A_{h}\left(t_{h}, \sigma_{h}\right),\left(s_{h}, \tau_{h}\right)\right]
\end{aligned}
$$

and hence

$$
\begin{aligned}
& {\left[B_{h}\left(s_{h}, \tau_{h}\right), u_{h}-v_{h}\right]} \\
& =\left[A(t, \sigma)-A\left(t_{h}, \sigma_{h}\right),\left(s_{h}, \tau_{h}\right)\right]+\left[B\left(s_{h}, \tau_{h}\right), u-v_{h}\right]
\end{aligned}
$$

for all $\left(\left(s_{h}, \tau_{h}\right), v_{h}\right) \in X_{h} \times M_{h}$. Then, the discrete inf-sup condition for $B_{h}$ (cf. (3.3)) leads to

$$
\begin{aligned}
& \beta^{*}\left\|u_{h}-v_{h}\right\| \\
& \quad \leq \sup _{0 \neq\left(s_{h}, \tau_{h}\right) \in X_{h}} \frac{\left[A(t, \sigma)-A\left(t_{h}, \sigma_{h}\right),\left(s_{h}, \tau_{h}\right)\right]+\left[B\left(s_{h}, \tau_{h}\right), u-v_{h}\right]}{\left\|\left(s_{h}, \tau_{h}\right)\right\|} .
\end{aligned}
$$

In this way, applying the Lipschitz continuity of $A_{1}$ and the boundedness of $B_{1}$ and $B$, we conclude from the above inequality that

$$
\left\|u_{h}-v_{h}\right\| \leq \frac{1}{\beta^{*}}\left(\gamma+\left\|B_{1}\right\|\right)\left\|(t, \sigma)-\left(t_{h}, \sigma_{h}\right)\right\|+\frac{\|B\|}{\beta^{*}}\left\|u-v_{h}\right\|
$$

for all $v_{h} \in M_{h}$. Therefore, estimates (4.28) and (4.26) complete the proof of the theorem

To end this section, we remark that the proof of our main Theorem 4.1 follows directly from Theorems 4.4 and 4.5 . 


\section{Application to coupling of mixed-FEM and BEM}

We now show an example of an exterior nonlinear boundary value problem, whose weak formulation, via the coupling of mixed-FEM and BEM, can be written in the dual-dual type form studied in the previous sections (see [9]).

Let $\Omega_{0}$ be a bounded and simply connected domain in $\mathbb{R}^{2}$ with Lipschitz continuous boundary $\Gamma_{0}$. Also, let $\Omega_{1}$ be the annular domain bounded by $\Gamma_{0}$ and another Lipschitz continuous closed curve $\Gamma_{1}$ whose interior region contains $\Omega_{0}$. In addition, let $a_{i}: \Omega_{1} \times \mathbb{R}^{2} \rightarrow \mathbb{R}(i=1,2)$ be nonlinear mappings satisfying certain appropriate conditions. Then, given $f_{1} \in L^{2}\left(\Omega_{1}\right)$, we consider the exterior nonlinear transmission problem

Find $u_{1} \in H^{1}\left(\Omega_{1}\right)$ and $u_{2} \in H_{\text {loc }}^{1}\left(\mathbb{R}^{2} \backslash \bar{\Omega}_{0} \cup \bar{\Omega}_{1}\right)$ such that

$$
\left.\begin{array}{rlrl}
u_{1} & =0 & & \text { on } \Gamma_{0} \\
-\sum_{i=1}^{2} \frac{\partial}{\partial x_{i}} a_{i}\left(\cdot, \nabla u_{1}(\cdot)\right) & =f_{1} & & \text { in } \Omega_{1} \\
u_{1}=u_{2}, \sum_{i=1}^{2} a_{i}\left(\cdot, \nabla u_{1}(\cdot)\right) n_{i}-\frac{\partial u_{2}}{\partial n} & =0 & & \text { on } \Gamma_{1} \\
-\Delta u_{2} & =0 & & \text { in } \mathbb{R}^{2} \backslash \bar{\Omega}_{0} \cup \bar{\Omega}_{1} \\
u_{2}(x) & =O(1) & & \text { as }\|x\| \rightarrow+\infty
\end{array}\right\}
$$

where $n=\left(n_{1}, n_{2}\right)$ denotes the unit outward normal to $\partial \Omega_{1}$.

This kind of problem appears in the computation of magnetic fields of electromagnetic devices (see, e.g., $[13,14]$ ), in some subsonic flow and fluid mechanics problems (see, e.g., [3, 4]), and also in steady heat conduction.

In what follows, we apply a dual-mixed finite element method in $\Omega_{1}$ and a Dirichlet-to-Neumann mapping (arising from the boundary integral equation method) in the exterior region $\mathbb{R}^{2} \backslash \bar{\Omega}_{0} \cup \bar{\Omega}_{1}$. To this end, we first introduce a sufficiently large circle $\Gamma$ with center at the origin such that its interior region contains $\bar{\Omega}_{0} \cup \bar{\Omega}_{1}$. We denote by $\Omega_{2}$ the annular region bounded by $\Gamma_{1}$ and $\Gamma$ and put $\Omega=\Omega_{1} \cup \Gamma_{1} \cup \Omega_{2}$. Then, we define the global unknowns $u$, the flux variable $\sigma$ and the global data $f$ as

$$
u=\left\{\begin{array}{ll}
u_{1} & \text { in } \Omega_{1} \\
u_{2} & \text { in } \Omega_{2}
\end{array} \quad \sigma=\left\{\begin{array}{ll}
\left(a_{1}(\cdot, \nabla u), a_{2}(\cdot, \nabla u)\right)^{T} & \text { in } \Omega_{1} \\
\nabla u & \text { in } \Omega_{2}
\end{array} \quad f= \begin{cases}f_{1} & \text { in } \Omega_{1} \\
0 & \text { in } \Omega_{2}\end{cases}\right.\right.
$$

respectively, and introduce the auxiliary unknowns

$$
\left.\begin{array}{l}
\theta=\nabla u \text { in } \Omega \\
\xi=\left.u\right|_{\Gamma}
\end{array}\right\}
$$

By applying the boundary integral equation method in the region exterior to the circle $\Gamma$, and according to the results from $[8,15]$, we obtain the Dirichlet-to-Neumann 
mapping $\frac{\partial u}{\partial \nu}=-2 W\left(\left.u\right|_{\Gamma}\right)$ on $\Gamma$ or, equivalently, $\sigma \cdot \nu=-2 W(\xi)$ on $\Gamma$ where $\nu$ is the unit outward normal to $\Gamma$ and $W$ is the hypersingular boundary integral operator associated to the Laplacian. Hence, it is not difficult to see that the exterior transmission problem (5.1) can be reformulated as the following non-local boundary value problem in $\bar{\Omega}$ :

Find $(\theta, \xi, \sigma, u) \in\left[L^{2}(\Omega)\right]^{2} \times H_{0}^{1 / 2}(\Gamma) \times H_{0}(\operatorname{div}, \Omega) \times L^{2}(\Omega)$ such that

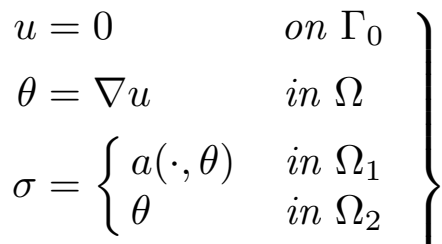

$$
\begin{aligned}
& \operatorname{div} \sigma=-f \quad \text { in } \Omega \\
& \sigma \cdot \nu=-2 W(\xi), u=\xi \quad \text { on } \Gamma)
\end{aligned}
$$

where we have adopted the notations

$$
\begin{aligned}
a(\cdot, \theta) & =\left(a_{1}(\cdot, \theta), a_{2}(\cdot, \theta)\right)^{T} \\
H_{0}^{1 / 2}(\Gamma) & =\left\{\lambda \in H^{1 / 2}(\Gamma):\langle\lambda, 1\rangle=0\right\} \\
H_{0}(\operatorname{div}, \Omega) & =\{\tau \in H(\operatorname{div}, \Omega):\langle 1, \tau \cdot \nu\rangle=0\}
\end{aligned}
$$

and hereafter $\langle\cdot, \cdot\rangle$ denotes the duality pairing of $H^{1 / 2}(\Gamma)$ and $H^{-1 / 2}(\Gamma)$ with respect to the $L^{2}(\Gamma)$-inner product.

Now, for the weak formulation we multiply the second equation in (5.3) by a function $\tau \in H_{0}(\operatorname{div}, \Omega)$, integrate by parts in $\Omega$ and use that $u=0$ on $\Gamma_{0}$ and $u=\xi$ on $\Gamma$ to obtain

$$
-\int_{\Omega} \theta \cdot \tau d x+\langle\xi, \tau \cdot \nu\rangle-\int_{\Omega} u \operatorname{div} \tau d x=0 .
$$

Next, the third equation in (5.3) is tested against $\zeta \in\left[L^{2}(\Omega)\right]^{2}$, which gives

$$
\int_{\Omega_{1}} a(\cdot, \theta) \cdot \zeta d x+\int_{\Omega_{2}} \theta \cdot \zeta d x-\int_{\Omega} \sigma \cdot \zeta d x=0 .
$$

Finally, the fourth and fifth equations in (5.3) are tested against $v \in L^{2}(\Omega)$ and $\lambda \in H_{0}^{1 / 2}(\Gamma)$, respectively, which yields

$$
\begin{aligned}
-\int_{\Omega} v \operatorname{div} \sigma d x & =\int_{\Omega} f v d x \\
2\langle\lambda, W(\xi)\rangle+\langle\lambda, \sigma \cdot \nu\rangle & =0 .
\end{aligned}
$$

Therefore, collecting appropriately (5.4) - (5.7) and putting $t=(\theta, \xi), X_{1}=\left[L^{2}(\Omega)\right]^{2}$ $\times H_{0}^{1 / 2}(\Gamma), M_{1}=H_{0}(\operatorname{div}, \Omega), X=X_{1} \times M_{1}$ and $M=L^{2}(\Omega)$, we arrive at the following variational formulation of problem (5.3): 
Find $((t, \sigma), u) \in X \times M$ such that

$$
\left[\begin{array}{cc}
A & B^{*} \\
B & O
\end{array}\right]\left[\begin{array}{c}
(t, \sigma) \\
u
\end{array}\right]=\left[\begin{array}{l}
\mathcal{F} \\
\mathcal{G}
\end{array}\right]
$$

with $\mathcal{F} \equiv O \in X^{\prime}$

$$
A(t, \sigma):=\left[\begin{array}{cc}
A_{1} & B_{1}^{*} \\
B_{1} & O
\end{array}\right]\left[\begin{array}{c}
t \\
\sigma
\end{array}\right] \in X^{\prime}=X_{1}^{\prime} \times M_{1}^{\prime}
$$

and where $A_{1}: X_{1} \rightarrow X_{1}^{\prime}, B_{1}: X_{1} \rightarrow M_{1}^{\prime}, B_{1}^{*}: M_{1} \rightarrow X_{1}^{\prime}, B: X \rightarrow M^{\prime}, B^{*}: M \rightarrow$ $X^{\prime}$ and $\mathcal{G} \in M^{\prime}$ are defined as

$$
\begin{aligned}
{\left[A_{1}(t), s\right] } & =\int_{\Omega_{1}} a(\cdot, \theta) \cdot \zeta d x+\int_{\Omega_{2}} \theta \cdot \zeta d x+2\langle\lambda, W(\xi)\rangle \\
{\left[B_{1}(t), \tau\right] } & =-\int_{\Omega} \theta \cdot \tau d x+\langle\xi, \tau \cdot \nu\rangle \\
{\left[B_{1}^{*}(\sigma), s\right] } & =-\int_{\Omega} \sigma \cdot \zeta d x+\langle\lambda, \sigma \cdot \nu\rangle \\
{[B(t, \sigma), v] } & =-\int_{\Omega} v \operatorname{div} \sigma d x \\
{\left[B^{*}(u),(s, \tau)\right] } & =-\int_{\Omega} u \operatorname{div} \tau d x \\
{[\mathcal{G}, v] } & =\int_{\Omega} f v d x
\end{aligned}
$$

for all $s=(\zeta, \lambda) \in X_{1}$, all $\tau \in M_{1}$ and all $v \in M$.

Clearly, because of the coefficients $a_{i}(i=1,2), A_{1}$ becomes a nonlinear operator. We note also that, assuming standard growth conditions on $a_{i}$ (see, e.g., $[8,10]$ ) one can easily prove that $A_{1}$ is strongly monotone and Lipschitz continuous, as required in Theorem 2.3. Moreover, it is not difficult to show that the variational formulation (5.8) verifies all the hypotheses of Theorem 2.4. In addition, it is possible to define explicit finite element subspaces of Raviart-Thomas type, so that all the hypotheses of Theorems 3.2 and 4.1 are satisfied. Furthermore, with this particular choice of subspaces one obtains that $V_{h}$ is included in $V$ and hence the usual Cea estimate holds. We omit further comments at this point and just mention that all the details are given in [9].

As a concluding remark we emphasize that, on the contrary to the previous procedures dealing with nonlinear transmission problems (via the coupling of mixedFEM and BEM) (see, e.g., [10, 11]), the present dual-dual approach does provide completely satisfactory results for both the continuous and discrete schemes. 


\section{References}

[1] Barrenechea, G. R., Gatica, G. N. and G. C. Hsiao: Weak solvability of interior transmission problems via mixed finite elements and Dirichlet-to-Neumann mappings. J. Comp. \& Appl Math. 100 (1998), 145 - 160.

[2] Brezzi, F. and M. Fortin: Mixed and Hybrid Finite Element Methods. New York, Berlin, Heidelberg: Springer-Verlag 1991.

[3] Feistauer, M.: Mathematical and numerical study of nonlinear problems in fluid mechanics. In: Proc. Conf. Equadiff 6, Brno 1985 (eds.: J. Vosmansky and M. Zlámal). Berlin: Springer-Verlag 1986, pp. $3-16$.

[4] Feistauer, M.: On the finite element approximation of a cascade flow problem. Num. Math. 50 (1987), $655-684$.

[5] Gatica, G. N.: Combination of mixed finite element and Dirichlet-to-Neumann methods in nonlinear plane elasticity. Appl. Math. Letters 10 (1997)6, 29 - 35.

[6] Gatica, G. N.: An application of Babuška-Brezzi's theory to a class of variational problems. Appl. Anal. 75 (2000), 297 - 303.

[7] Gatica, G. N. and N. Heuer: A dual-dual formulation for the coupling of mixed-FEM and BEM in hyperelasticity. SIAM J. Num. Anal. 38 (2000), $380-400$.

[8] Gatica, G. N. and G. C. Hsiao: The uncoupling of boundary integral and finite element methods for nonlinear boundary value problems. J. Math. Anal. Appl. 189 (1995), 442 $-461$.

[9] Gatica, G.N. and S. Meddahi: A dual-dual mixed formulation for nonlinear exterior transmission problems. Math. Comp. 70 (2001), 1461 - 1480.

[10] Gatica, G. N. and W. L. Wendland: Coupling of mixed finite elements and boundary elements for linear and nonlinear elliptic problems. Appl. Anal. 63 (1996), 39 - 75.

[11] Gatica, G. N. and W. L. Wendland: Coupling of mixed finite elements and boundary elements for a hyperelastic interface problem. SIAM J. Num. Anal. 34 (1997), 2335 2356 .

[12] Girault, V. and P.-A. Raviart: Finite Element Methods for Navier-Stokes Equations. Theory and Algorithms. Berlin, Heidelberg, New York, Tokyo: Springer-Verlag 1986.

[13] Heise, B.: Nonlinear field calculations with multigrid Newton methods. Impact Comp. in Sci. \& Eng. 5 (1993), 75 - 110.

[14] Heise, B.: Analysis of a fully discrete finite element method for a nonlinear magnetic field problem. SIAM J. Num. Anal. 31 (1994), 745 - 759.

[15] Hsiao, G. C. and S. Zhang: Optimal order multigrid methods for solving exterior boundary value problems. SIAM J. Num. Anal. 31 (1994), 680 - 694.

[16] Le Tallec, P.: Existence and approximation results for nonlinear mixed problems. Application to incompressible finite elasticity. Num. Math. 38 (1982), 365 - 382.

[17] Le Tallec, P. and V. Ruas: On the convergence of the bilinear velocity-constant pressure finite method in viscous flow. Comp. Methods in Appl. Mech. \& Eng. 54 (1986), 235 $-243$.

[18] Necas, J.: Introduction to the Theory of Nonlinear Elliptic Equations. Chichester, New York, Brisbane, Toronto, Singapore: John Wiley \& Sons 1986.

[19] Scheurer, B.: Existence et approximation de point selles pour certain problemes non linéaires. R.A.I.R.O. Anal. Num. 11 (1977), 369 - 400. 
782 G. N. Gatica

Received 07.11.2000 